\title{
Basaloid/blue salivary gland tumors
}

\author{
Raja R Seethala \\ Department of Pathology, University of Pittsburgh Medical Center, Pittsburgh, PA, USA
}

\begin{abstract}
Basaloid tumors are a common diagnostic problem in salivary gland pathology. However, delineating each of these tumor types is facilitated by an algorithmic approach incorporated by tumor border and cell types. This approach greatly diminishes the challenge of separating polymorphous low-grade adenocarcinoma (PLGA) from adenoid cystic carcinoma (ACC). Despite the overlap in growth pattern, ACC is biphasic while PLGA is not. More relevant challenges, namely differentiation of the biphasic basaloid neoplasms including: epithelial-myoepithelial carcinoma (EMCA), cellular pleomorphic adenoma (PA), basal cell adenoma (BCA), and basal cell adenocarcinoma (BCAC), are resolved by a combination of morphologic, immunophenotypic, and to a limited extent, molecular features. Among the most challenging scenarios is high-grade transformation of any of the aforementioned entities. Here, the diagnosis requires recognition of a conventional component and exclusion of metastatic (or in some cases primary) SCC and even select neuroendocrine carcinomas and sarcomas in some cases.

Modern Pathology (2017) 30, S84-S95; doi:10.1038/modpathol.2016.190
\end{abstract}

\section{Introduction}

Basaloid or 'blue' salivary gland tumors comprise a significant proportion of all tumors encountered in salivary gland, primary, metastatic as well as nonsalivary-type lesions. Some tumors are fairly characteristic and easily diagnosed, whereas others pose significant diagnostic challenges, and occasionally unconventional strategies are required to establish more exotic diagnoses. This review covers the spectrum of basaloid salivary gland tumors from basic to advanced or elite diagnoses.

\section{Discussion}

Basaloid Tumors 001—Applications of Salivary Tumor Basics

As with all salivary gland tumors, basaloid salivary gland tumors can be approached using an algorithm that takes into consideration border, color, and phenotype. ${ }^{1}$ Although salivary gland tumors do have considerable overlap in the type of matrix, tinctorial, characterstics and cellularity at low power, they do have tendencies to cluster into either basaloid, clear

Correspondence: Dr RR Seethala, MD, Department of Pathology, University of Pittsburgh Medical Center, A614.X Presbyterian University Hospital, 200 Lothrop Street, Pittsburgh, PA 15217, USA.

E-mail: seethalarr@upmc.edu

Received 1 July 2016; revised 29 September 2016; accepted 29 September 2016 cell, or oncocytoid categories as depicted in the Venn diagram in Figure 1a. It must be noted, however, that although prototypical examples of salivary tumors fit nicely into this schema, variant morphologies may deviate towards the center of the diagram. Notable examples relevant to basaloid tumors include epithelial-myoepithelial carcinoma, classically a clear cell tumor, and (metastatic) squamous cell carcinoma, particularly when it is non-keratinizing. Acinic cell carcinoma is 'blue' at low power magnification (Figure 1b) but technically distinct from the other basaloid tumors. The classic blue appearance is a result of the basophilic zymogen granules (hence the moniker 'blue dot tumor'). In contrast, truly basaloid tumors have the blue appearance as a result of their cellularity and high nuclear to cytoplasmic ratio, which recapitulates a primitive basal cell, as demonstrated in the myoepithelioma in Figure 1c.

Another significant tool in salivary gland tumor diagnosis is tumor border assessment. In general, encapsulated/well demarcated, uninodular tumors as such are benign while highly infiltrative tumors are malignant. ${ }^{1}$ However, a significant proportion of tumors may demonstrate a multinodular configuration, which spans both benign and low-grade malignant entities and thus poses the most significant challenge. Also, within a category, variant morphologies (ie, basal cell adenoma tubulotrabecular vs membranous) may separate in terms of growth pattern. Of note, recurrent benign tumors may demonstrate a multinodular configuration, thus overlapping with many low-grade malignancies. 
a
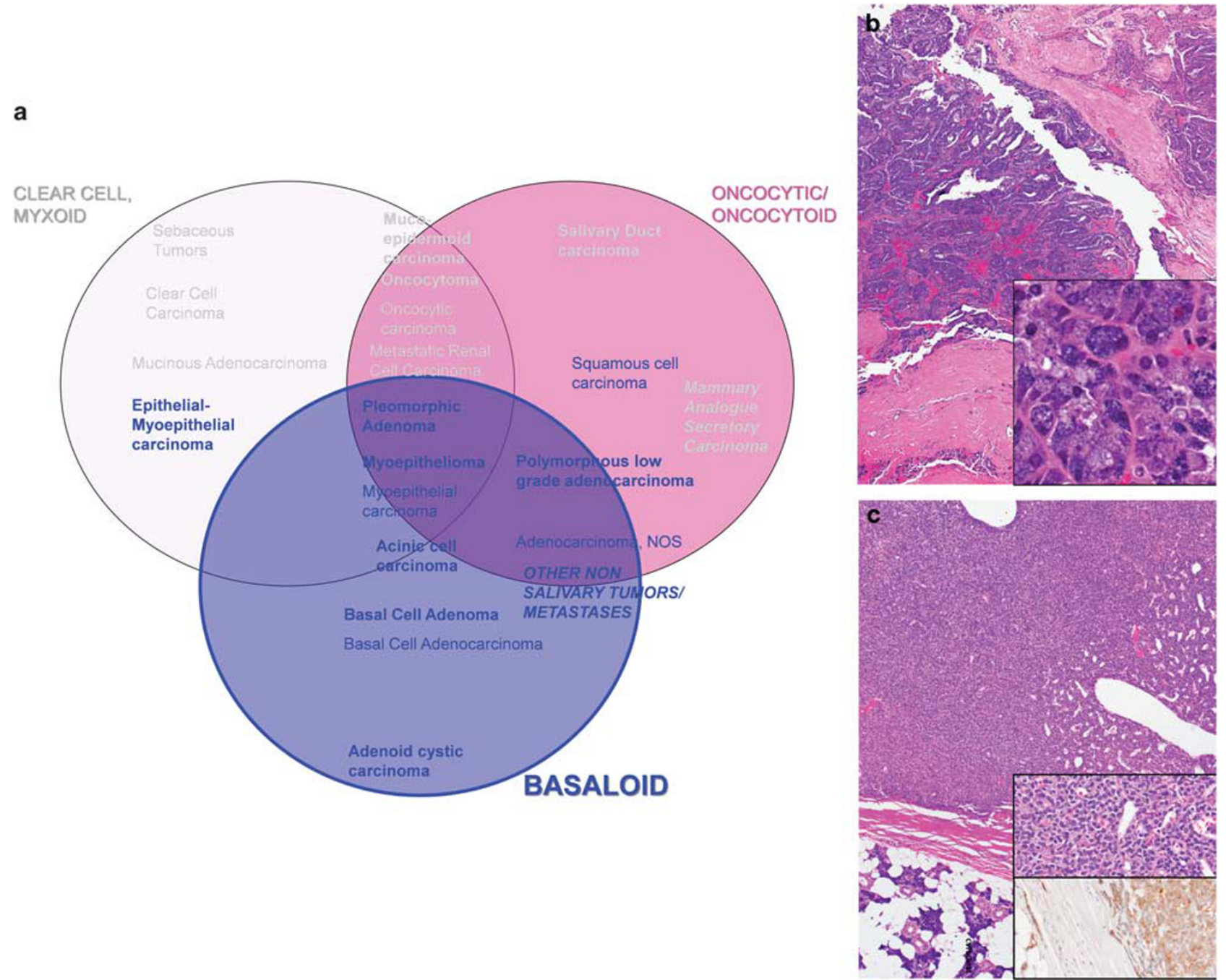

Figure 1 Overview of basaloid tumors. (a) A Venn diagram demonstrating relationships of major salivary gland tumor entities including metastases. Blue circle encompasses the classic basaloid/blue tumor types. Blue font encompasses classic basaloid entities as well as traditionally clear cell or oncocytoid entities that can manifest as basaloid tumors. (b) Acinic cell carcinoma is blue mainly because of its zymogen granules (inset) and is not strictly speaking basaloid. (c) This myoepithelial carcinoma, however, is basaloid in the traditional sense demonstrating scant cytoplasm and a high nuclear to cytoplasmic ratio (top inset). This tumor is diffusely calponin positive (bottom inset).

Metastases and non-salivary-type basaloid tumors may span a spectrum of growth patterns and may actually be deceptively well demarcated if they involve a lymph node and are subsequently delimited by the nodal capsule. As many of the basaloid tumors show morphologic overlap in terms of cytonuclear features and stroma, border assessment is critical in distinguishing infiltrative tumors such as adenoid cystic carcinoma (ACC) from other mimics. This also highlights a significant limitation of salivary gland biopsies, as these often do not contain adequate tumor normal interface to assess border (Figure 2).

Although low power features such as border and phenotype narrow the differential diagnosis tremendously, the final steps to further resolve a diagnosis requires a higher magnification survey of cytonuclear features to define cell phenotype. Tumors resemble various components of the ductoacinar unit. It is important to note that this description is one of morphogenesis, ie, 'what a tumor is,' rather than histogenesis, ie, 'where a tumor comes from,' as the exact location of tumor stem cells along the ductoacinar unit is unclear. In other words, the origin of acinic cell carcinoma is not necessarily from acinar cells, all that is known (and readily visible by light microscopy) is that it is tumor that shows acinar differentiation. Biphasic salivary gland tumors are unique in that they recapitulate the bilayer of the intercalated and striated ducts and demonstrate sharply demarcated abluminal myoepithelial and/or basal cells and luminal ductal cells. This arrangement is integral to the tumor and can be accentuated by immunohistochemical staining. The 

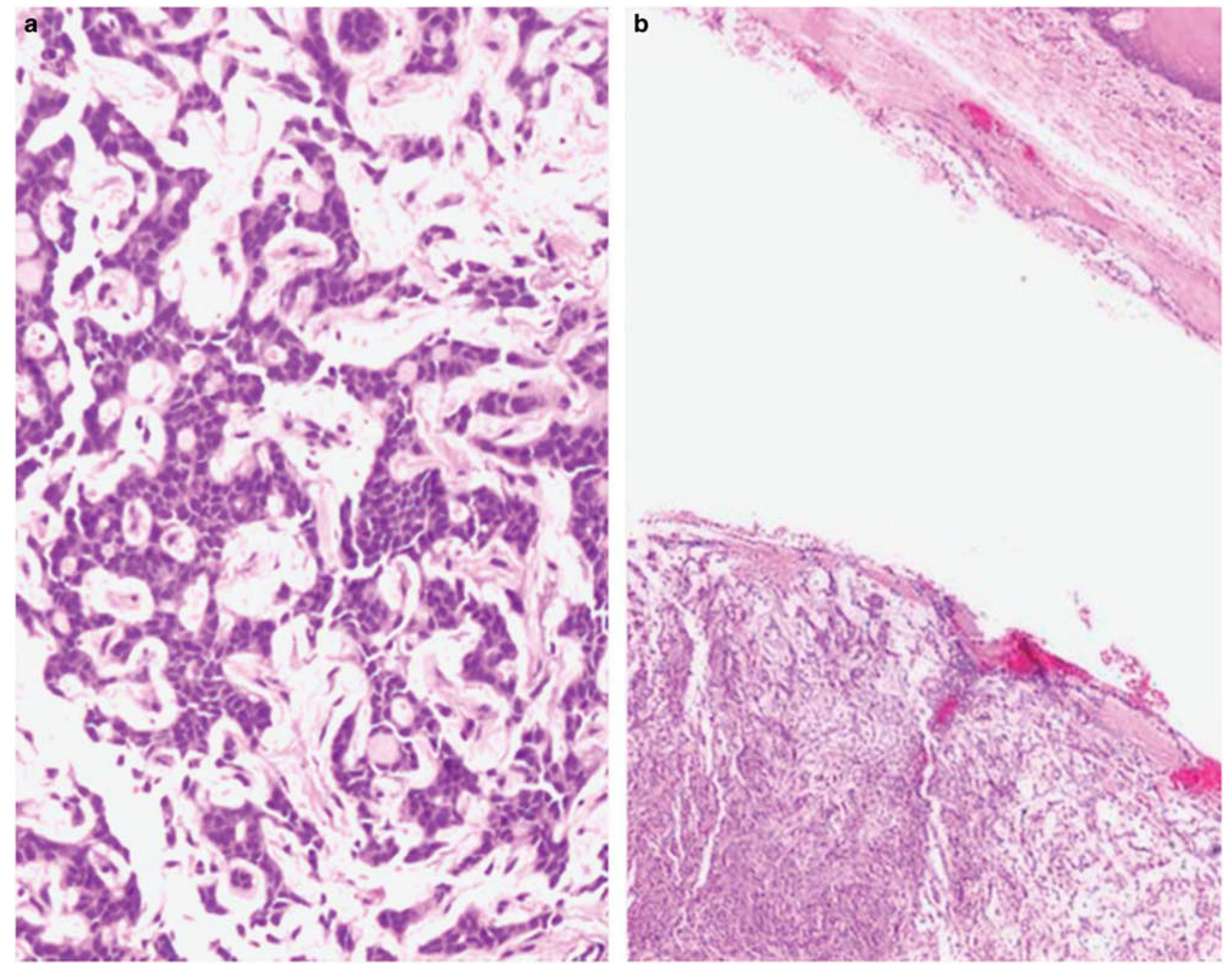

Figure 2 Importance of tumor border. (a) This tumor demonstrates adenoid cystic-like areas and was in fact diagnosed as adenoid cystic carcinoma on biopsy. (b) However, the resection demonstrates a well-delineated interface and cells streaming into a myxoid stroma more in keeping with a pleomorphic adenoma. This patient was disease-free for at least 23 years.

concept of biphasic salivary gland tumors is a major theme within the basaloid salivary gland tumor differential diagnosis.

\section{Basaloid Tumors 101: Polymorphous Low-Grade Adenocarcinoma vs Adenoid Cystic Carcinoma: Still a Challenge?}

Although previous descriptions of polymorphous low-grade adenocarcinoma (PLGA) existed, ie, terminal duct carcinoma ${ }^{2}$ and lobular carcinoma, ${ }^{3}$ the term PLGA was coined in 1984 by Evans and Batsakis. ${ }^{4}$ It describes a series of low-grade yet infiltrative tumors that resembled adenoid cystic carcinoma from an architectural standpoint. PLGA predominate in the oral cavity, specifically palate, and despite their propensity to infiltrate and show perineural invasion, they generally have a favorable outcome; although they may show locoregional recurrence in about one-third of cases, distant metastases and death from disease are exceptionally rare. ${ }^{5-7}$ Histologically, PLGA is composed of monomorphic cells in ovoid vesicular nuclei and scant-tomodest amounts of lightly eosinophilic cytoplasm arrayed in targetoid, tubular or fascicular growth patterns, though cribriform, solid, and papillary growth patterns may also be noted. ${ }^{5}$ Thus at low power, PLGA is often 'blue' or basaloid, but does show some overlap with oncocytoid or 'pink tumors.' ${ }^{1}$ PLGA may produce myxohyaline matrix similar to that of adenoid cystic carcinoma and other basaloid salivary gland tumors. Cribriform adenocarcinoma of salivary gland, also described as cribriform adenocarcinoma of tongue (CAT) and cribriform adenocarcinoma of minor salivary gland (CAMSG) was originally described in the base of the tongue. ${ }^{8,9}$ These tumors resemble classic PLGA, but with a more cribriform-to-papillary glomeruloid growth pattern, more pronounced nuclear clearing, and a higher propensity for lymphovascular invasion 

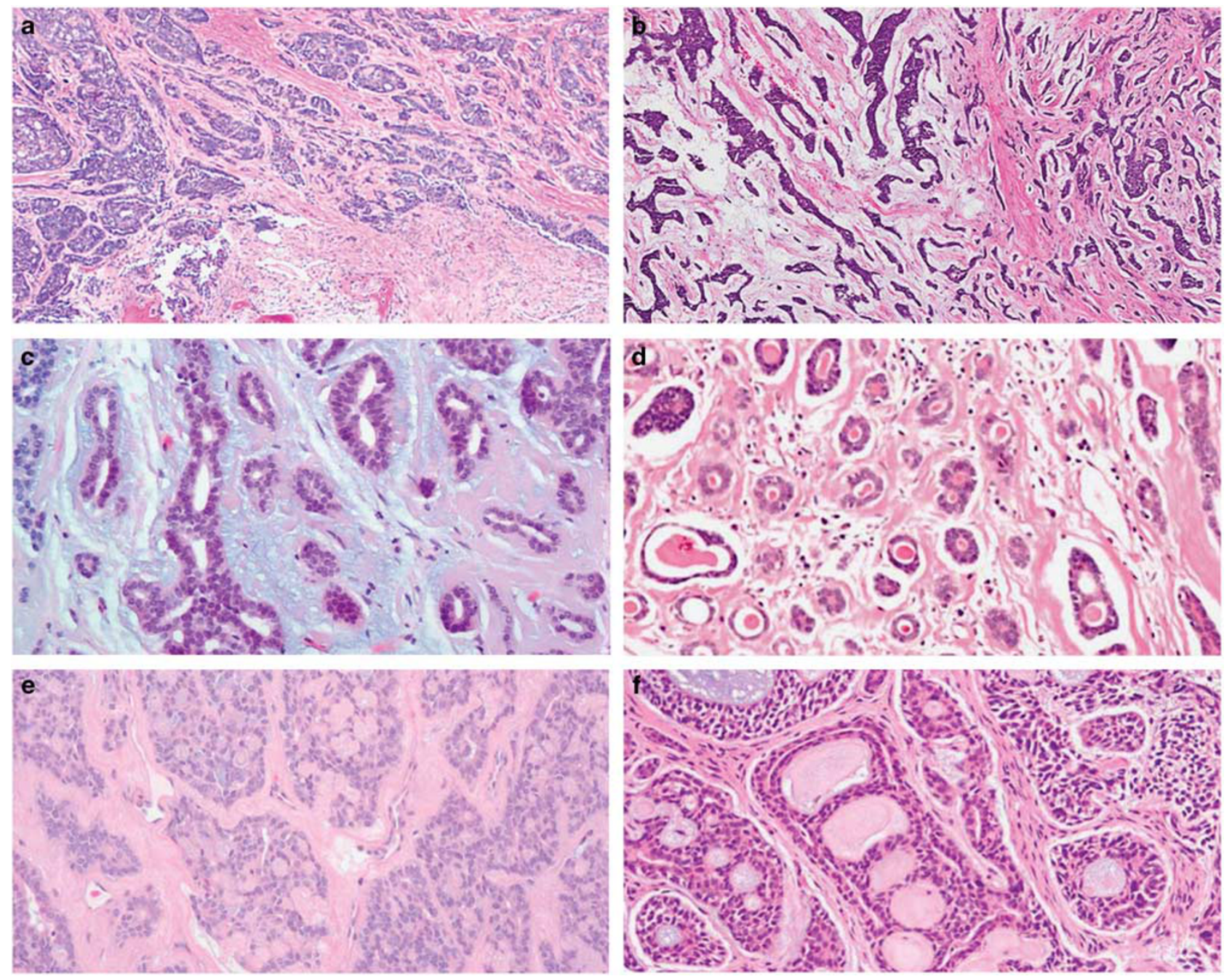

Figure 3 Shared growth patterns between PLGA and ACC. (a) PLGA and (b) ACC both demonstrate infiltrative borders. Tubular and cribriform patterns may be noted in both PLGA (c and e), as well as ACC (d and f).

and nodal metastasis. Whether CAMSG is justifiably a separate entity or a more aggressive variant of PLGA is currently a topic of controversy and beyond the scope of this review. Furthermore, both morphologies will pose similar challenges in terms of differential diagnosis.

Historically, the far more aggressive adenoid cystic carcinoma (ACC) has been the main differential diagnostic consideration for PLGA given their similarities in growth pattern (Figures 3a-f). However, despite the numerous publications and immunohistochemical studies addressing this differential diagnosis, this problem is actually quite simple to resolve when applying the basic approach noted above. Despite similarities in the growth pattern, architecture, and stromal characteristics, PLGA is not a biphasic tumor, unlike ACC. This key concept is what trivializes this perceived challenge. PLGA is polymorphous in pattern, but fairly monomorphic from a cellular perspective. It is fundamentally composed of a ductal cell type. ACC of all basic patterns is a biphasic tumor with abluminal myoepithelial cells and luminal ductal cells. In fact, this key also distinguishes PLGA from the other basaloid tumors that will be discussed later such as pleomorphic adenoma, epithelial-myoepithelial carcinoma, and basal cell adenocarcinoma, all of which are biphasic. ${ }^{1}$

Perhaps the second most important distinguishing feature of PLGA is its cytonuclear features. Tumor cells are monomorphic, with variable amounts of eosinophilic cytoplasm and demonstrate characteristically ovoid vesicular nuclei with inconspicuous, peripherally placed nucleoli. ${ }^{5}$ CASG nuclear morphology is more extreme and is often likened to papillary thyroid carcinoma. In contrast, both the myoepithelial and ductal components of ACC consist of angulated hyperchromatic nuclei, though this is more pronounced in the myoepithelial component. Cytoplasm is extremely scant in comparison with PLGA, thus imparting a 'bluer' appearance at low power ${ }^{1}$ (Figures $4 \mathrm{a}$ and $\mathrm{b}$ ). 

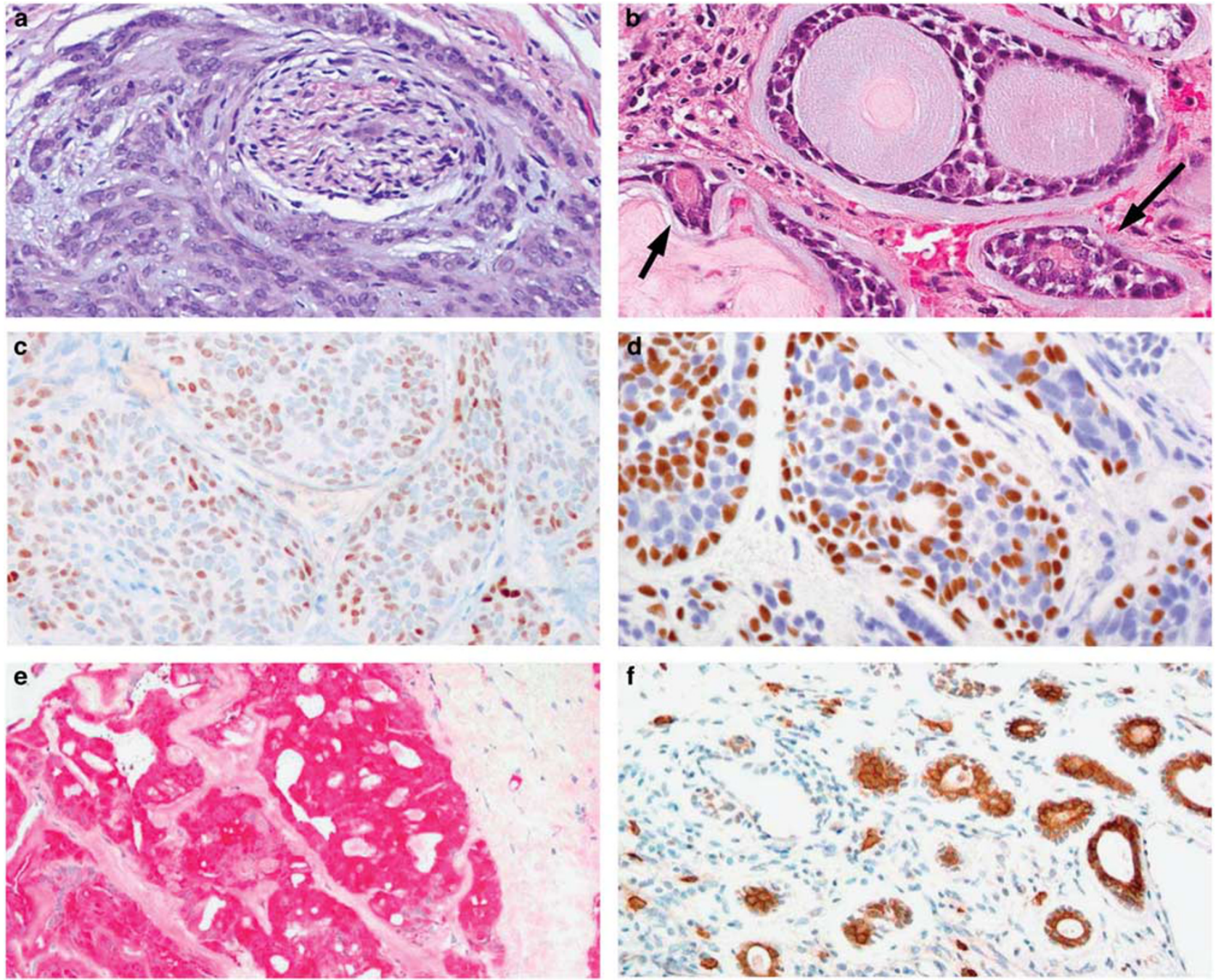

Figure 4 Distinguishing features between PLGA and ACC. (a) PLGA consists of one cell type and demonstrates ovoid vesicular nuclei with variably cleared chromatin. (b) ACC on the other hand consists of angulated hyperchromatic nuclei, and is biphasic with abluminal myoepithelial cells and luminal ductal cells (arrows). Not the clefting of tumor nests from the adjacent stroma. (c) PLGA is monophasic, whereas p63 may show heterogeneous non-patterned staining as seen here. (d) In contrast, ACC consistently demonstrates positivity, but more importantly, it is in a patterned abluminal distribution in keeping with its biphasic nature. (e) S100 is much stronger in PLGA, while (f) C-kit is much stronger in ACC favoring the luminal ductal components.

Thus under optimal conditions (good fixation, adequate sampling), the challenge of distinguishing PLGA from ACC is fairly low level. However, this is not to make light of the difficulties that may be encountered with limited sampling (often the case in actual practice), which may impair resolution of whether a tumor is biphasic. Also, suboptimal or non-standard fixation (ie, decalcification solution) may result in some effacement of chromatin, thus imparting a more vesicular appearance to the nuclei of an ACC, thus mimicking PLGA. In addition, as ACC approach a mucosal surface, they can truly deviate morphologically from the angulated hyperchromatic appearance with more abundant cytoplasm and columnar cell change in the ductal component.
Thus in these scenarios, immunohistochemical studies may be helpful in resolving this differential diagnosis. The approach can be divided into two tiers. The first tier is to establish whether or not the tumor in question is biphasic. Generally, PLGA stains with ductal type markers (ie, low molecular weight keratins), and are mostly negative for myoepithelial or basal markers. ACC on the other hand shows a dual staining pattern intrinsic to its biphasic nature. Luminal ductal cells tend to be positive for the low molecular weight keratins while the abluminal myoepithelial cells are positive for muscle markers and p63 (Figures 4c and d). Thus if biphasic morphology is not readily noted by routine light microscopy, it can be accentuated by the application of a low molecular weight keratin and a basal or myoepithelial marker. 
Recently, however, p63 has been given some attention in this differential diagnosis. This is regarded as a basal/myoepithelial marker. Traditionally, p63 is expected to be negative in PLGA, ${ }^{10}$ but more recent studies have noted frequent and diffuse positivity in PLGA. ${ }^{11,12}$ In this author's personal experience, this change is likely a result of the more frequent use of autostainers with enhanced epitope retrieval in comparison with earlier studies, many of which used manual methods. Nonetheless, this may pose a diagnostic dilemma if this pitfall is not realized. One solution to this is the utilization of the $\Delta$ Np63 isoform-specific antibody $\mathrm{p} 40$, which is a more specific basal/myoepithelial marker. ${ }^{11,12}$ PLGA are, to date, uniformly negative for $\mathrm{p} 40$, whereas the basal/myoepithelial cells of ACC will still be positive. However, even without $\mathrm{p} 40$, there are differences in the staining pattern for p63 in PLGA and ACC that are easily recognized. PLGA shows a patchy to diffuse staining with heterogeneity in intensity (ie, non-patterned) while ACC still shows the characteristic biphasic staining pattern with uniform intensity.

The second tier is to use markers that are preferentially expressed in PLGA or ACC. The traditional marker S100 is actually very robust as it stains PLGA far more intensely and diffusely than ACC (Figure 4e), and outperforms other markers such as C-kit and CD43. C-kit is commonly used as it is preferentially expressed in ACC (mainly the ductal component) in comparison with PLGA ${ }^{13}$ (Figure 4f). Here it must be noted that PLGA can be positive albeit in a weaker, more heterogeneous manner. ${ }^{14}$ Proliferation index can also fit under this approach. As ACC is more proliferative than PLGA, proliferation index (ie, using MIB1) may supplement other markers, particularly on biopsies. ${ }^{15}$ Recently, MYB protein immunohistochemistry shows promise as it serves as a surrogate for MYB-NFIB translocation and other $M Y B$ alterations that now define a large proportion of ACC, ${ }^{16}$ and PLGA are expected to be negative and are instead characterized by alterations in the PRKD family of genes. ${ }^{17}$ Distinction between PLGA and ACC is summarized in Table 1.

\section{Basaloid Tumors 400: Biphasic Basaloid Tumors: The Real Everyday Challenge}

When delving below the surface of basaloid salivary gland tumor diagnosis, it becomes apparent that many of the tumors are actually biphasic, and their distinction poses challenge from both a routine and consultative perspective. In addition to ACC, the biphasic basaloid salivary gland tumors that will be discussed here include cellular pleomorphic adenoma (PA), epithelial-myoepithelial carcinoma (EMCA), basal cell adenoma (BCA), and basal cell adenocarcinoma (BCAC). The focus here will be on EMCA, BCA and BCAC as they are less common.

EMCA is, as its name suggests, a prototypical biphasic low-grade salivary gland malignancy. Formally described by Donath et al in $1972,{ }^{18}$ this is a tumor that predominates in the sixth decade with a slight female predilection (3:2). Site distribution is as follows: parotid (60-80\%), submandibular gland (8$15 \%)$, sinonasal $(5-10 \%)$, and other sites including larynx, trachea, and lower respiratory tract. Overall outcome is favorable with a 5 year survival of $\sim 94 \% .{ }^{19}$ The characteristic growth pattern consists of a multinodular permeative border with variable hyaline sclerosis. Classically this is actually a clear cell tumor consisting of abluminal clear polygonal myoepithelial cells and compressed tubules with scant eosinophilic cytoplasm. However cytoplasmic tinctorial variant morphologies (ie, oncocytic) exist, and when EMCA become more cellular, they can enter the basaloid salivary tumor differential diagnosis. EMCA may have overgrowth of either component, which may mask the biphasic nature by light microscopy. As expected, EMCA will stain in a biphasic manner showing luminal positivity for low molecular weight keratins and abluminal cell positivity for muscle markers, p63, and high molecular weight keratins. S100 may stain either the ductal or myoepithelial component.

EMCA do show a good deal of overlap with the other biphasic tumors. EMCA can resemble cellular PA, but importantly, it is distinguished by its multinodular infiltrative growth and sclerosis (Figure 5b). Cellular PA may show sclerosis, but do not infiltrate. In addition, the abluminal myoepithelial cells are smaller, less distinct, and characteristically 'stream' into a chondromyxoid stroma (Figure 5a). EMCA may also overlap with tubular patterned ACC. However, ACC is more infiltrative, and composed of more angulated hyperchromatic nuclei with scant cytoplasm. In addition, ACC tubules will cleft from the basement membrane. EMCA may show cell dyshesion, but is between the ductal and myoepithelial cell layer rather than the

Table 1 Discriminatory features between PLGA and ACC

\begin{tabular}{lll}
\hline & PLGA & ACC \\
\hline Morphology & Monophasic, ovoid vesicular nuclei & Biphasic, angulated hyperchromatic nuclei with stromal clefting \\
P63/P40 & Heterogenous non-patterned/negative & Abluminal patterned/abluminal patterned \\
S100 & Strongly diffusely positive & Weakly focally positive \\
C-kit & Negative to weakly positive & Moderately to strongly positive luminal pattern \\
Molecular features & PRKD alterations & MYB alterations \\
\hline
\end{tabular}



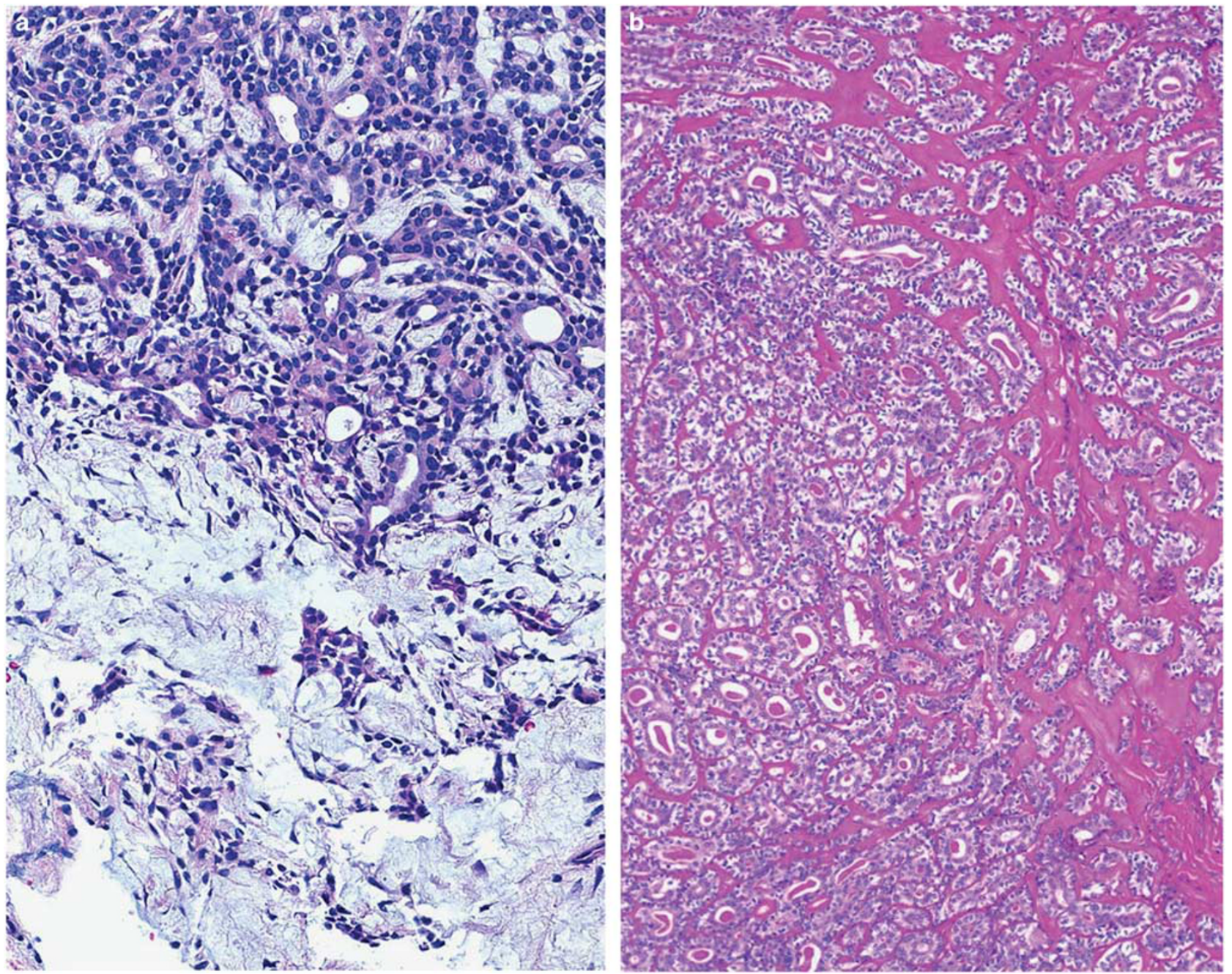

Figure 5 Distinction between EMCA and cellular PA. (a) Cellular PA is biphasic and bilayered but well demarcated, and the myoepithelial cells often stream into a chondromyxoid stroma as seen here. (b) EMCA is more characteristically multinodular and tends to show more plump polygonal abluminal myoepithelial cells with bilayered tubules surrounded by sclerotic stroma rather than myxoid stroma.

basement membrane. Immunoprofile of both tumor types shows considerable overlap, including c-kit reactivity. From a molecular standpoint, ACC are defined by $M Y B$ alterations, ${ }^{16}$ while EMCA often show RAS mutations. ${ }^{20}$

BCA is a rare tumor with a female predilection (2:1), and are historically designated as monomorphic adenomas. Almost all BCA arise in the parotid gland with submandibular gland being a distant second; other sites are exceptionally rare. Histologically, BCA can demonstrate several growth patterns including: tubulotrabecular, cribriform, solid, and membranous, making the term monomorphic adenoma a misnomer (Figures 6a-d). ${ }^{21}$ Two of these subtypes are rather distinctive: the membranous (or dermal analog) type, and tubulotrabecular types. Membranous BCA are unique in that they can be multifocal and associated with Brooke Spiegler syndrome. As such, even sporadic tumors show CYLD1 mutations and loss. ${ }^{22,23}$ These tumors have a much higher rate of local recurrence $(25 \%)$ than other BCA ( $2 \%$ recurrence rate) likely stemming from their propensity for multifocality. Tubulotrabecular BCA are more common and often have CTNNB1 mutations. ${ }^{24-26}$

In addition to diverse growth patterns, BCA demonstrates diverse cell types arranged in bilayered manner typical of biphasic tumors and outer layer peripheral palisading is the hallmark of all BCA subtypes. With tubulotrabecular BCA in particular, the outer or abluminal layers can be subdivided further into a peripheral dark cell layer and adjacent pale cell layer, making it arguably a 'triphasic' tumor (Figures 7a-d). The dark cell layer shows peripheral palisading, scant cytoplasm, and dark but still vesicular nuclei. The overlying pale cell layer shows larger more vesicular nuclei, and more abundant eosinophilic, often squamoid cytoplasm. Central to these cell layers is the luminal cell layer, which consists of small cuboidal cells with 

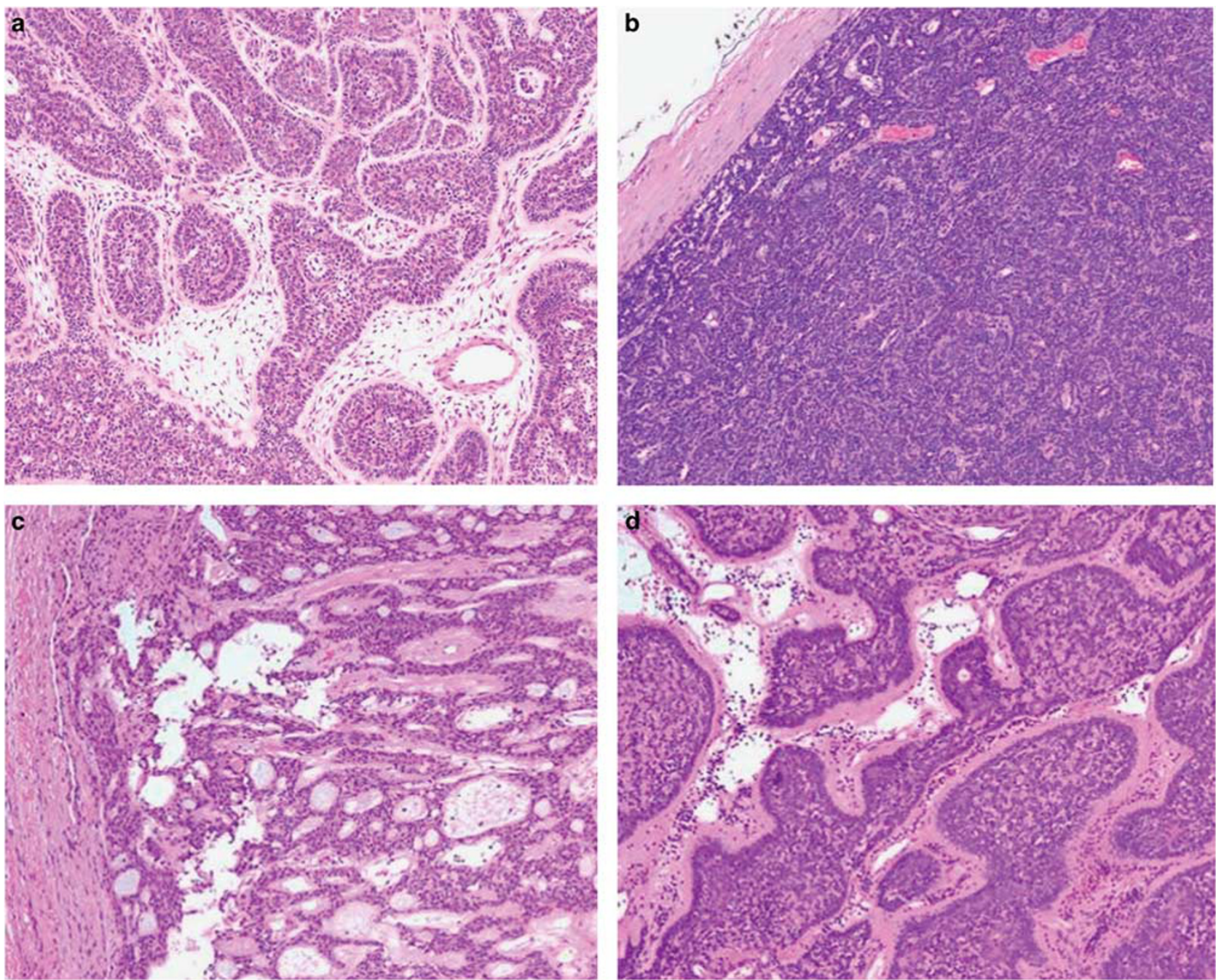

Figure 6 Patterns in BCA. (a) Tubulotrabecular, (b) solid, (c) cribriform, (d) membranous. All the patterns show some degree of peripheral palisading of the outermost abluminal cell layer, which can be visualized at low power particularly in (a and d).

scant eosinophilic cytoplasm, forming compressed tubules. Mature squamous and sebaceous elements may be present in all types of BCA. The tubulotrabecular BCA have varying degrees of a peculiar spindled myxoid stroma that has been shown ultrastructurally to be myoepithelial. ${ }^{1}$ Immunohistochemical stains recapitulate the triphasic morphology. The outermost dark cell layer is actually myoepithelial and is positive for both muscle makers and basal markers such as p63 and high molecular weight keratins. The overlying pale abluminal cells are basal only, with p63 and high molecular weight keratin expression. The luminal cell layers stain only with lower molecular weight keratins. S100 is variable among all the cell populations including the ductal cells, but is intensely positive in the myoepithelial-derived stroma present in some tubulotrabecular BCA (Figure 7e). The presence of CTTNB1 mutations in BCA result in nuclear $\beta$-catenin accumulation that can be demonstrated by immunohistochemistry (Figure 7f).
Tubulotrabecular BCA also express LEF-1 (Figure $7 \mathrm{~g})^{27}$ which is activated and in response to nuclear accumulation of $\beta$-catenin, and then in turn transactivates a variety of Wnt-responsive elements (Figure 7C). Membranous BCA do not usually express nuclear $\beta$-catenin, though LEF-1 reactivity may still be noted, likely a result of a Wntindependent role.

BCA can overlap with all basaloid biphasic salivary tumors, but once a benign configuration is established, the main distinction is between BCA and cellular PA. BCA with prominent myoepithelialderived stroma in particular can mimic cellular PA. However, unlike cellular PA, the stroma in a BCA does not blend into the epithelial nests, and instead remains distinct. Nuclear $\beta$-catenin can also help distinguish these two.

BCAC is the malignant counterpart of BCA. BCAC present about one decade later than BCA and have an even gender distribution, though site distribution is essentially the same. As hinted by the later 

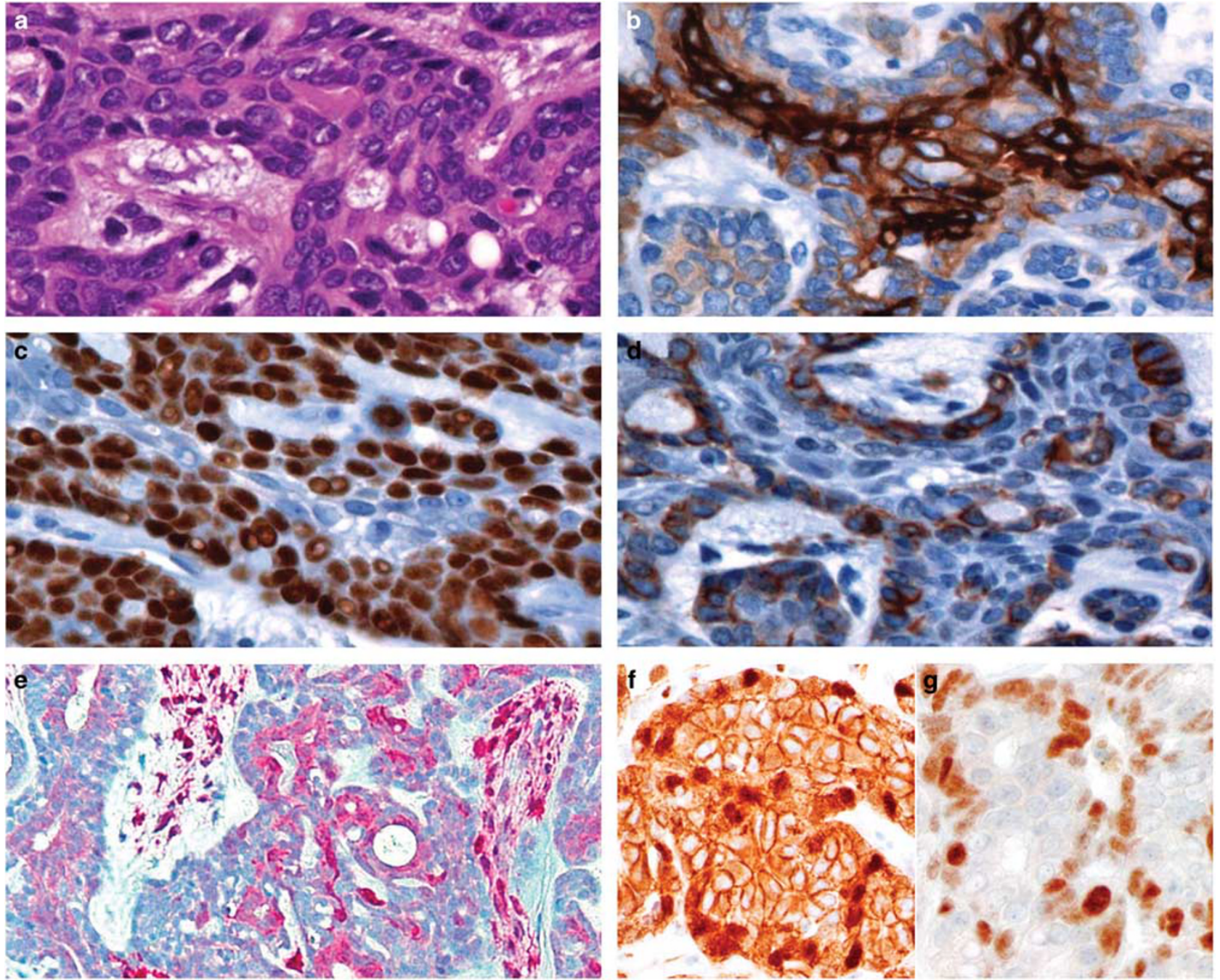

Figure 7 Immunoprofile of BCA. (a) The trabecular BCA pattern is not only biphasic but arguably triphasic. The abluminal cell layer can be subdivided on light microscopy. The peripheral layer is palisaded and hyperchromatic or 'dark.' The abluminal cell layer above this dark peripheral layer is more vesicular or 'pale' and has somewhat more eosinophilic cytoplasm. The innermost layer is luminal and ductal as demonstrated by the compressed tubule in the center. (b) The keratin stain shows ductal luminal accentuation, most prominent in the inner cell layer with diminishing staining towards the abluminal cell layers. (c) The p63 stain demonstrates positivity in both the dark and pale abluminal cell layers and is negative in the central luminal ductal layer. (d) Smooth muscle actin is only positive in the dark abluminal cell layer indicating that this layer has a true myoepithelial phenotype. (e) S100 highlights the peculiar myoepithelial-derived stroma common to tubulotrabecular patterned BCA. Note that the ductal cells are also weakly positive here. (f) This tubular BCA shows abluminal dark layer nuclear accumulation of $\beta$-catenin, and (g) LEF-1.

presentation, some BCAC likely arise from BCA, but most are captured de novo. The membranous type of BCA has the highest propensity for malignant transformation to BCAC. Indeed membranous and solid patterns are the most common patterns seen in BCAC, though all the patterns seen in BCA are possible. ${ }^{28,29}$ The molecular phenotype of BCAC, is less studied. Recently PIK3CA mutations and biallelic inactivation of NFKBIA have been described in BCAC. One case with $C Y L D 1$ deletion was detected as well, but no cases demonstrated CTNNB1 mutations to date. ${ }^{25,26}$ Recently a t(1;3)(q21,p21) translocation has been described in a BCAC as well. ${ }^{30}$ Overall, these findings may suggest that the pathogenesis for most BCAC is actually distinct from most BCA.

BCAC are most readily distinguished from BCA by their invasive growth, which is typically multinodular and only occasionally frankly infiltrative. Perineural invasion is documented in about onethird of cases. Other helpful but not required features include an elevated mitotic count ( $>4$ mitoses per 10 h.p.f.), and a ki-67 proliferation index of $>5 \%{ }^{31}$ As a rule, BCAC show a low cytonuclear grade; only rare examples show high-grade morphology.

The biphasic nature and multinodular growth pattern are similar to that seen in EMCA, particularly that of a tubulotrabecular BCAC. However, BCAC 
still retain the prominent peripheral palisading, and do not often show clear cytoplasm in the abluminal cells as seen in EMCA. Although EMCA abluminal cells can be multilayered, they are not as organized (ie, biphasic but not triphasic) as the tubulotrabecular BCAC. Along these lines, although both BCAC and EMCA have a myoepithelial component, in EMCA all abluminal cells are myoepithelial, whereas in BCAC the outermost dark layer is myoepithelial while the overlying pale layer is purely basal. Thus in EMCA, the distribution of p63 positive cells is roughly similar to that of another myoepithelial marker (ie, calponin, actin). In contrast, in BCAC, p63 is positive in both dark and light abluminal layers, whereas muscle markers are only positive in the dark layer. A more obvious discriminatory marker on the other hand is the nuclear $\beta$-catenin and LEF-1, when present, usually in the tubulotrabecular variant. On the other hand, membranous and cribriform BCAC may be mistaken for ACC. ACC, however, is more frankly infiltrative. In contrast to the small hyaline droplets of membranous BCAC, cribriform ACC has larger hyaline cylinders. Although both tumors show dark nuclei, those of ACC are angulated while those of BCAC are still somewhat vesicular, and the outer layer shows palisading. Finally, while ACC is a relatively 'pure' tumor without other elements, BCAC often show squamous and sebaceous elements. The main use of immunohistochemistry is to establish a triphasic pattern in BCAC. A marker such as c-kit is expressed in both the tumor types. It must also be noted that MYB immunohistochemical staining may show focal positivity in BCA and BCAC.

\section{Basaloid Tumors 1337: Pleomorphic Basaloid Tumors and Beyond}

As difficult as it may be to distinguish between the entities previously discussed, pleomorphic basaloid tumors offer added layers of complexity. In addition to primary salivary gland tumors, non-salivary and even non-epithelial neoplasms enter the differential diagnosis. In general, it is unusual for a primary salivary gland basaloid tumor to show pleomorphic cytonuclear features, thus necessitating a high index of suspicion for metastasis.

However, primary pleomorphic basaloid salivary tumors exist, and usually represent high-grade transformation (HGT) of another low-grade tumor type, which not surprisingly, is often basaloid to begin with. $^{32}$ ACC, EMCA, acinic cell carcinoma, and PLGA/CASG represent basaloid tumor types that are documented to undergo HGT. ${ }^{32-37}$ As this designation implies, these variants behave more aggressively than their purely conventional counterparts. Surprisingly, the transformed components for all these tumor types looks remarkably similar, with ductal components predominating and demonstrating the appearance of a high-grade adenocarcinoma, or undifferentiated carcinoma. In general, the biphasic nature in HGT is lost, but it can be retained in EMCA. ${ }^{36}$ HGT in any tumor type is often accompanied by necrosis and desmoplasia (Figure 8). As the morphology can be non-descript, the most reliable method to establish diagnosis is recognition of the conventional component.

The differential diagnoses for basaloid salivary gland tumors with HGT includes salivary duct

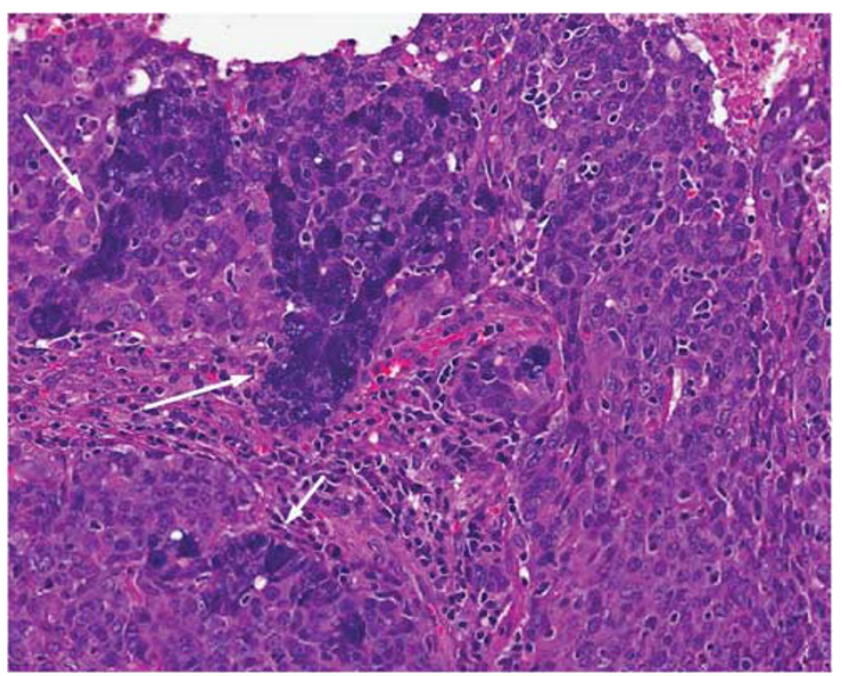

Figure 8 Acinic cell carcinoma with high-grade transformation. This is basically a solid undifferentiated carcinoma with interspersed acinar differentiation (arrows), which is the key to diagnosis here.

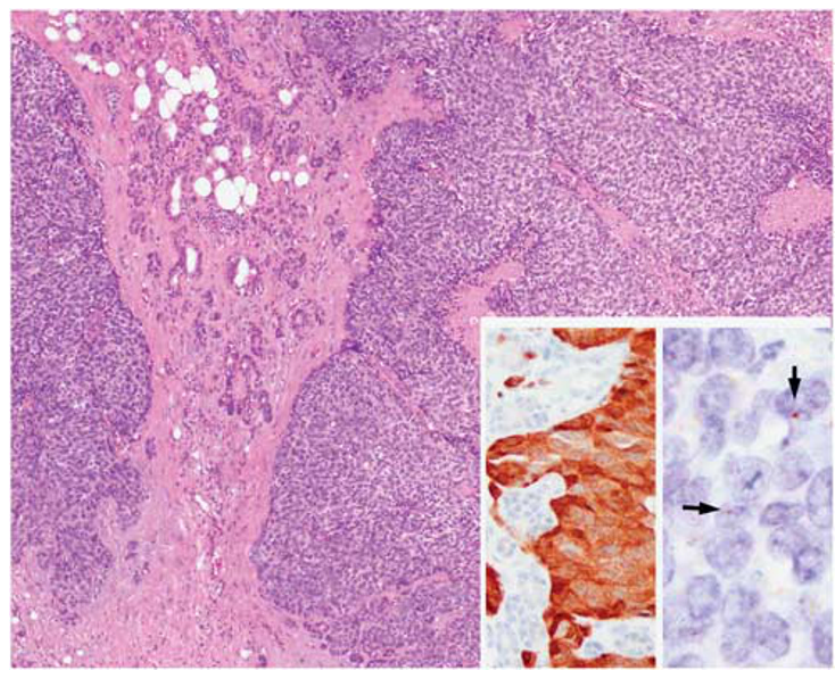

Figure 9 An HPV-related SCC of oropharynx metastasizing to parotid and mimicking a basaloid tumor. The high-grade morphology can be similar to that of a primary salivary gland carcinoma with high-grade transformation. However, this tumor showed no areas of a conventional salivary type carcinoma, thus raising suspicion for a metastasis. Insets: left-p16 is diffusely strongly positive, and right-in situ hybridization for HPV-DNA shows punctate staining (arrows). 
carcinoma, metastatic basaloid, and non-keratinizing squamous cell carcinoma (SCC), and non-salivary non-epithelial lesions. Salivary duct carcinoma may resemble any given basaloid tumor with HGT, as it is a pleomorphic adenocarcinoma that often shows necrosis and desmoplasia. The fundamental difference is that salivary duct carcinoma is an apocrine tumor and is thus 'pink' rather than blue. In addition, given this apocrine phenotype, it is characteristically androgen receptor (AR) positive. Most AR-negative tumors labeled as salivary duct carcinoma actually represent HGT of other entities, such as those noted above. ${ }^{38}$ Basaloid as well as non-keratinizing SCC can resemble salivary gland tumors ranging from ACC to BCAC. At minor salivary sites, primary basaloid or non-keratinizing SCC enters the differential diagnosis, while in parotid, metastasis enters the differential diagnosis. At primary sites, a surface or in situ component is a critical distinguishing factor since a primary salivary gland tumor is not expected to show this. When a metastasis is being considered, clinical history and immunohistochemical workup may be required. Notably non-keratinizing SCC metastases may masquerade as basaloid tumors (Figure 9). Rarely neuroendocrine carcinomas (metastases and rare primaries), as well as sarcomas (desmoplastic small round cell tumor, adamantinoma-like Ewing sarcoma, and biphasic synovial sarcoma) may enter the differential diagnosis. ${ }^{39}$ These are not usually intuitive initial diagnostic considerations, but warrant consideration if the typically salivary type entities are excluded, and the immunophenotype is not as expected.

\section{Summary}

Basaloid salivary gland tumors are diverse but can be stratified by border and cell types as with other salivary gland tumors. PLGA/CASG is not a biphasic tumor unlike ACC, rendering the distinction between these two entities fairly simple with adequate sampling. ACC, EMCA, cellular PA, BCA, and BCAC are all biphasic basaloid tumors with morphologic overlap but key discriminatory features. Most of these tumors can undergo HGT presenting as pleomorphic or high-grade carcinomas. Diagnosis rests on identification of a conventional component and exclusion of metastatic (or in some cases primary) SCC and occasionally, select neuroendocrine carcinomas and sarcomas.

\section{Disclosure/conflict of interest}

The author declares no conflict of interest.

\section{References}

1 Seethala RR. Diseases of the salivary gland. In: Wick MD, LiVolsi VA, Pfeifer JD, Stelow EB (eds).
Silverberg's Principles and Practice of Surgical Pathology and Cytopathology. Cambridge, UK: Cambridge University Press: Cambridge, UK, 2015, Vol 2, pp 1706-1803.

2 Batsakis JG, Pinkston GR, Luna MA, et al. Adenocarcinomas of the oral cavity: a clinicopathologic study of terminal duct carcinomas. J Laryngol Otol 1983;97: 825-835.

3 Freedman PD, Lumerman H. Lobular carcinoma of intraoral minor salivary gland origin. Report of twelve cases. Oral Surg Oral Med Oral Pathol 1983;56: 157-166.

4 Evans HL, Batsakis JG. Polymorphous low-grade adenocarcinoma of minor salivary glands. A study of 14 cases of a distinctive neoplasm. Cancer 1984;53: 935-942.

5 Castle JT, Thompson LD, Frommelt RA, et al. Polymorphous low grade adenocarcinoma: a clinicopathologic study of 164 cases. Cancer 1999;86:207-219.

6 Seethala RR, Johnson JT, Barnes EL, et al. Polymorphous low-grade adenocarcinoma: the University of Pittsburgh experience. Arch Otolaryngol Head Neck Surg 2010;136:385-392.

7 Evans HL, Luna MA. Polymorphous low-grade adenocarcinoma: a study of 40 cases with long-term follow up and an evaluation of the importance of papillary areas. Am J Surg Pathol 2000;24:1319-1328.

8 Skalova A, Sima R, Kaspirkova-Nemcova J, et al. Cribriform adenocarcinoma of minor salivary gland origin principally affecting the tongue: characterization of new entity. Am J Surg Pathol 2011;35:1168-1176.

9 Michal M, Skalova A, Simpson RH, et al. Cribriform adenocarcinoma of the tongue: a hitherto unrecognized type of adenocarcinoma characteristically occurring in the tongue. Histopathology 1999;35:495-501.

10 Seethala RR, LiVolsi VA, Zhang PJ, et al. Comparison of p63 and p73 expression in benign and malignant salivary gland lesions. Head Neck 2005;27:696-702.

11 Rooper L, Sharma R, Bishop JA. Polymorphous low grade adenocarcinoma has a consistent p63+/p40immunophenotype that helps distinguish it from adenoid cystic carcinoma and cellular pleomorphic adenoma. Head Neck Pathol 2015;9:79-84.

12 Owosho AA, Aguilar CE, Seethala RR. Comparison of p63 and p40 (DeltaNp63) as Basal, Squamoid, and Myoepithelial Markers in Salivary Gland Tumors. Appl Immunohistochem Mol Morphol 2015;24:501-508.

13 Holst VA, Marshall CE, Moskaluk CA, et al. KIT protein expression and analysis of c-kit gene mutation in adenoid cystic carcinoma. Mod Pathol 1999;12: 956-960.

14 Penner CR, Folpe AL, Budnick SD. C-kit expression distinguishes salivary gland adenoid cystic carcinoma from polymorphous low-grade adenocarcinoma. Mod Pathol 2002;15:687-691.

15 Skalova A, Simpson RH, Lehtonen H, et al. Assessment of proliferative activity using the MIB1 antibody help to distinguish polymorphous low grade adenocarcinoma from adenoid cystic carcinoma of salivary glands. Pathol Res Pract 1997;193:695-703.

16 Brill LB 2nd, Kanner WA, Fehr A, et al. Analysis of MYB expression and MYB-NFIB gene fusions in adenoid cystic carcinoma and other salivary neoplasms. Mod Pathol 2011;24:1169-1176.

17 Weinreb I, Piscuoglio S, Martelotto LG, et al. Hotspot activating PRKD1 somatic mutations in polymorphous low-grade adenocarcinomas of the salivary glands. Nat Genet 2014;46:1166-1169. 
18 Donath K, Seifert G, Schmitz R. Diagnosis and ultrastructure of the tubular carcinoma of salivary gland ducts. Epithelial-myoepithelial carcinoma of the intercalated ducts. Virchows Arch A Pathol Pathol Anat 1972;356:16-31.

19 Seethala RR, Barnes EL, Hunt JL. Epithelialmyoepithelial carcinoma: a review of the clinicopathologic spectrum and immunophenotypic characteristics in 61 tumors of the salivary glands and upper aerodigestive tract. Am J Surg Pathol 2007;31:44-57.

20 Chiosea SI, Miller M, Seethala RR. HRAS mutations in epithelial-myoepithelial carcinoma. Head Neck Pathol 2014;8:146-150.

21 Dardick I, Lytwyn A, Bourne AJ, et al. Trabecular and solid-cribriform types of basal cell adenoma. A morphologic study of two cases of an unusual variant of monomorphic adenoma. Oral Surg Oral Med Oral Pathol 1992;73:75-83.

22 Yu GY, Ubmuller J, Donath K. Membranous basal cell adenoma of the salivary gland: a clinicopathologic study of 12 cases. Acta Otolaryngol 1998;118:588-593.

23 Choi HR, Batsakis JG, Callender DL, et al. Molecular analysis of chromosome $16 q$ regions in dermal analogue tumors of salivary glands: a genetic link to dermal cylindroma? Am J Surg Pathol 2002;26:778-783.

24 Kawahara A, Harada H, Abe H, et al. Nuclear beta-catenin expression in basal cell adenomas of salivary gland. J Oral Pathol Med 2011;40:460-466.

25 Jo VY, Sholl LM, Krane JF. Distinctive patterns of CTNNB1 (beta-Catenin) alterations in salivary gland basal cell adenoma and basal cell adenocarcinoma. Am J Surg Pathol 2016;40:1143-1150.

26 Wilson TC, Ma D, Tilak A, et al. Next-generation sequencing in salivary gland basal cell adenocarcinoma and basal cell adenoma. Head Neck Pathol 2016;10: 494-500.

27 Bilodeau EA, Acquafondata M, Barnes EL, et al. A comparative analysis of LEF-1 in odontogenic and salivary tumors. Hum Pathol 2015;46:255-259.

28 Wilson TC, Robinson RA. Basal cell adenocarcinoma and basal cell adenoma of the salivary glands: a clinicopathological review of seventy tumors with comparison of morphologic features and growth control indices. Head Neck Pathol 2015;9:205-213.
29 Muller S, Barnes L. Basal cell adenocarcinoma of the salivary glands. Report of seven cases and review of the literature. Cancer 1996;78:2471-2477.

30 Saluja K, Rao PH, Myers JN, et al. Novel t(1;3)(q21,p21) translocation in a basal cell adenocarcinoma of the parotid gland: potential association with tumorigenesis. Hum Pathol 2016;54:189-192.

31 Nagao T, Sugano I, Ishida Y, et al. Basal cell adenocarcinoma of the salivary glands: comparison with basal cell adenoma through assessment of cell proliferation, apoptosis, and expression of p53 and bcl-2. Cancer 1998;82:439-447.

32 Costa AF, Altemani A, Hermsen M. Current concepts on dedifferentiation/high-grade transformation in salivary gland tumors. Pathol Res Int 2011;2011: 325965.

33 Nagao T, Gaffey TA, Serizawa H, et al. Dedifferentiated adenoid cystic carcinoma: a clinicopathologic study of 6 cases. Mod Pathol 2003;16:1265-1272.

34 Seethala RR, Hunt JL, Baloch ZW, et al. Adenoid cystic carcinoma with high-grade transformation: a report of 11 cases and a review of the literature. Am J Surg Pathol 2007;31:1683-1694.

35 Skalova A, Sima R, Vanecek T, et al. Acinic cell carcinoma with high-grade transformation: a report of 9 cases with immunohistochemical study and analysis of TP53 and HER-2/neu genes. Am J Surg Pathol 2009;33: 1137-1145.

36 Roy P, Bullock MJ, Perez-Ordonez B, et al. Epithelialmyoepithelial carcinoma with high grade transformation. Am J Surg Pathol 2010;34:1258-1265.

37 Simpson RH, Pereira EM, Ribeiro AC, et al. Polymorphous low-grade adenocarcinoma of the salivary glands with transformation to high-grade carcinoma. Histopathology 2002;41:250-259.

38 Williams L, Thompson LD, Seethala RR, et al. Salivary duct carcinoma: the predominance of apocrine morphology, prevalence of histologic variants, and androgen receptor expression. Am J Surg Pathol 2015;39:705-713.

39 Lezcano C, Clarke MR, Zhang L, et al. Adamantinomalike Ewing sarcoma mimicking basal cell adenocarcinoma of the parotid gland: a case report and review of the literature. Head Neck Pathol 2015;9:280-285. 